Supporting Information

\title{
Robust Yellow-Violet Pigments Tuned by Site-Selective Manganese Chromophores
}

Xiao Zhou, ${ }^{a}$ Jinjin Yang, ${ }^{a}$ Chuanhui Zhu, ${ }^{a}$ Yifeng Han, ${ }^{a}$ Zhongxiong Sun, ${ }^{a}$ Xiaokang Huang, ${ }^{a}$ Luyu Cao, ${ }^{a}$ Jing Wang, ${ }^{a}$ Yuanyuan Fang, ${ }^{b}$ Kai Wang, ${ }^{b}$ Bo Zou, ${ }^{b}$ Man-Rong $L i,{ }^{a, *}$

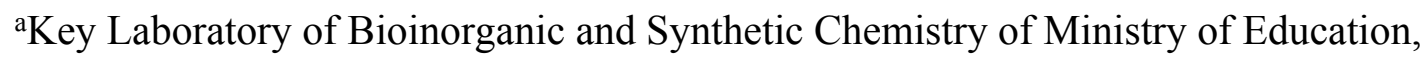
School of Chemistry, Sun Yat-Sen University, Guangzhou 510275, China.

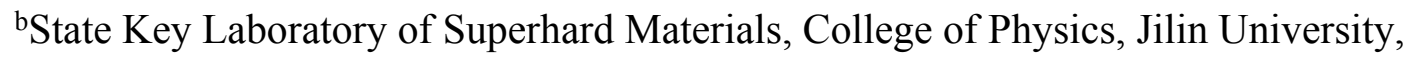
Changchun 130012, China.

*Email: limanrong@mail.sysu.edu.cn (M.-R. Li) 


\section{SUPPLEMENTARY FIGURES}
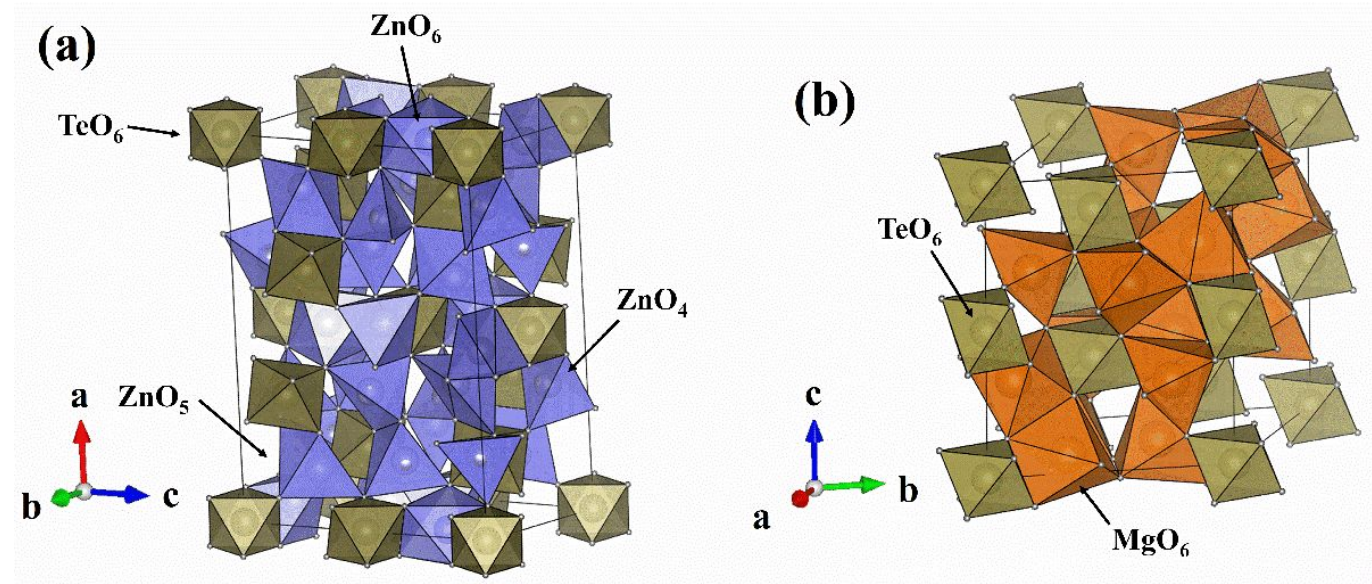

Figure S1. Polyhedral viewing of the crystal structure of (a) ZTO and (b) MTO hosts. 

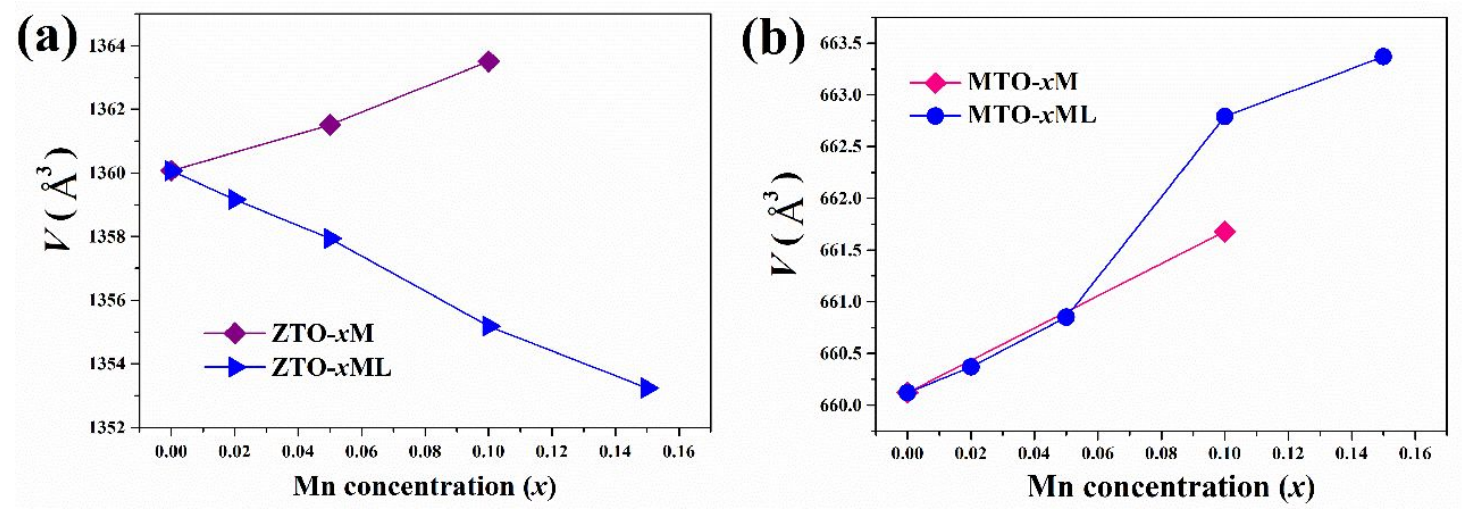

Figure S2. Unit cell volume evolution of (a) ZTO- $x \mathrm{M}$ and ZTO- $x \mathrm{ML}$, (b) MTO- $x \mathrm{M}$ and MTO-xML with different Mn and Mn-Li concentrations. 

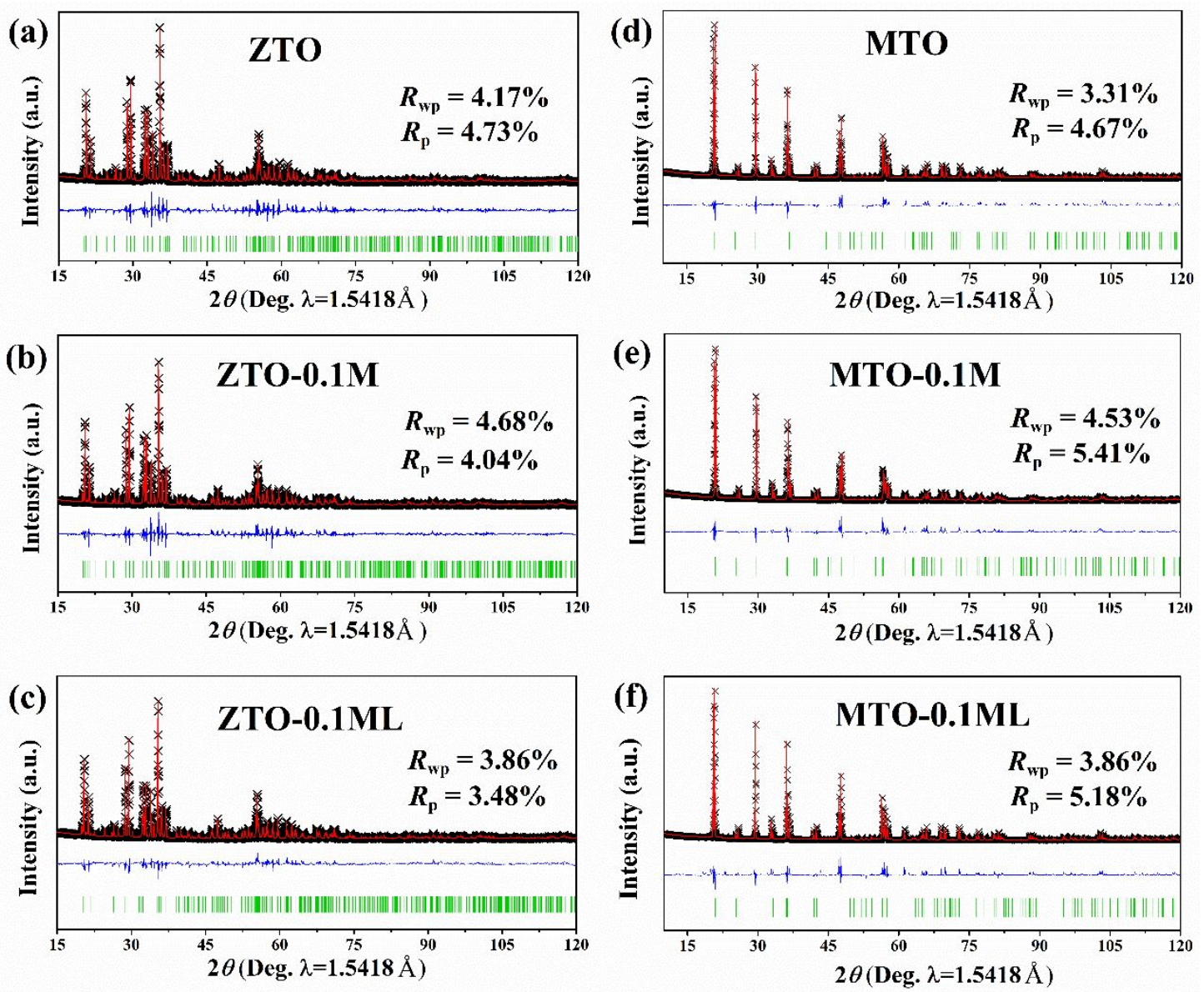

Figure S3. Rietveld refinements of PXD data of (a) ZTO, (b) ZTO-0.1M, (c) ZTO-

0.1ML, (d) MTO, (e) MTO-0.1M, and (f) MTO-0.1ML. Calculated (red line) and experimental (crosses) PXD patterns of sample. The green sticks stand for the positions of Bragg reflection and the blue line marks the difference between observed and calculated data. 


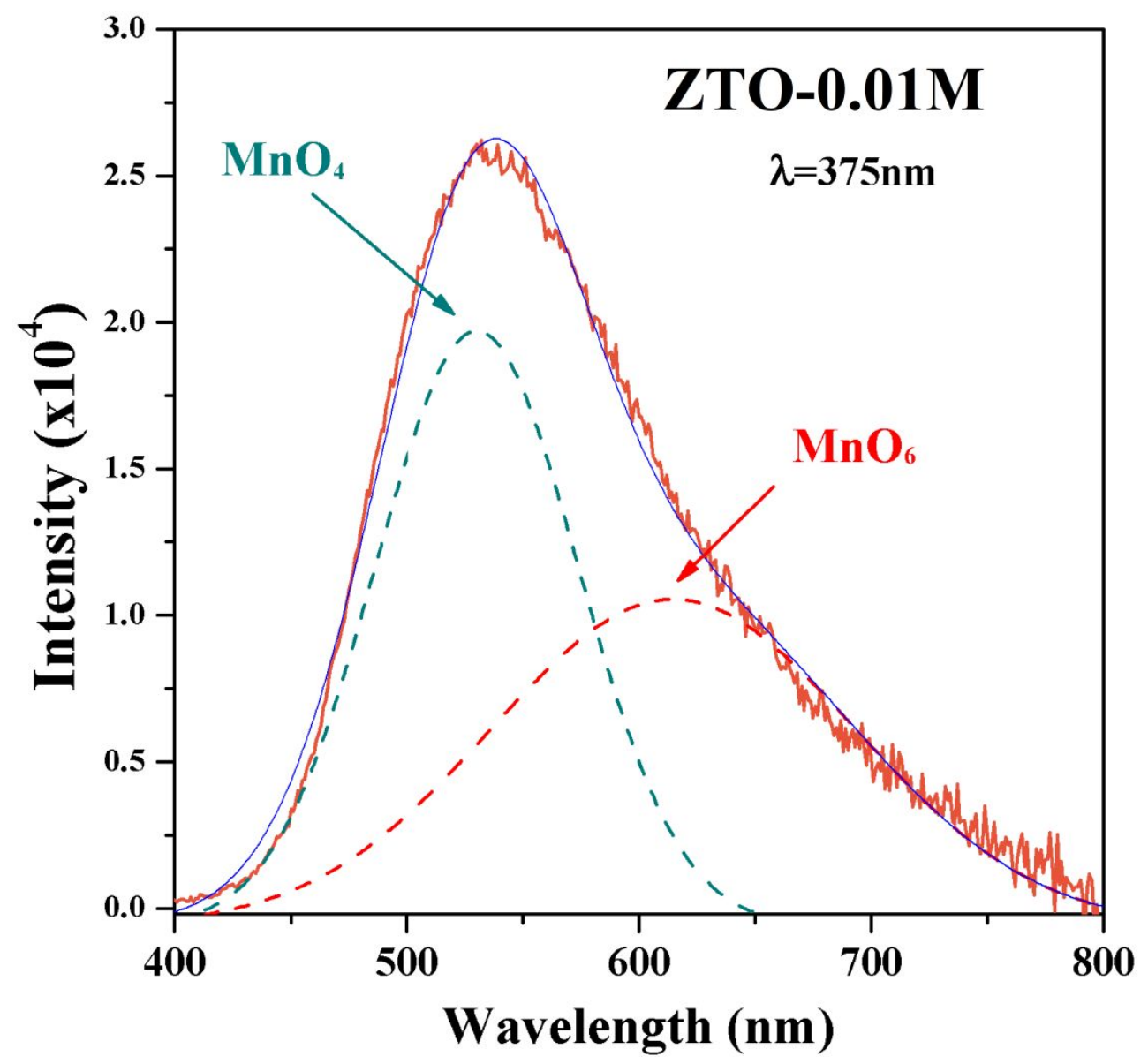

Figure S4. Photoluminescence spectrum of ZTO-0.01M. 


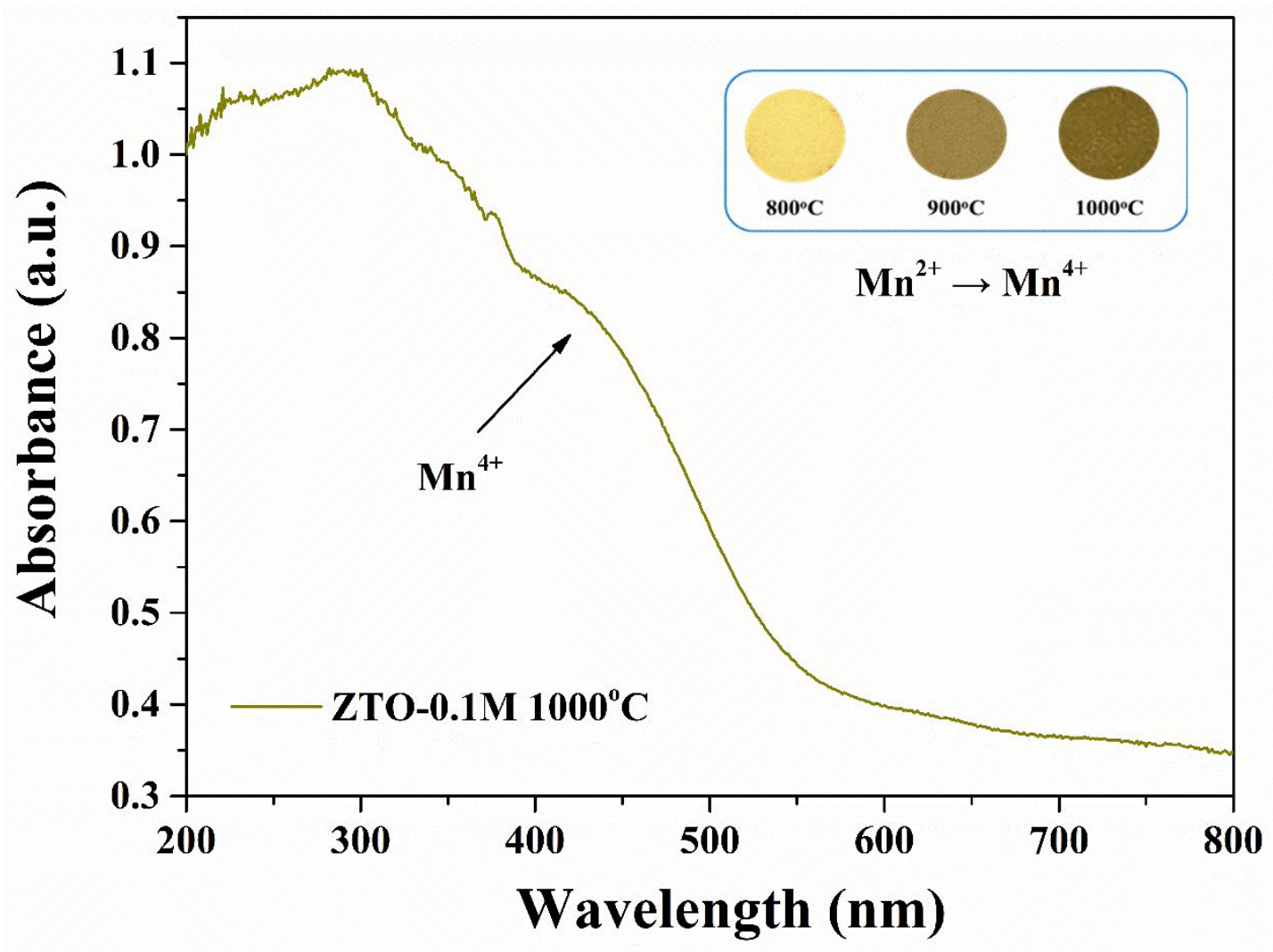

Figure S5. Absorption spectra of ZTO- $0.1 \mathrm{M}$ synthesized at $1000^{\circ} \mathrm{C}$, the inset is the color photo of ZTO-0.1M after examined at different temperatures. 


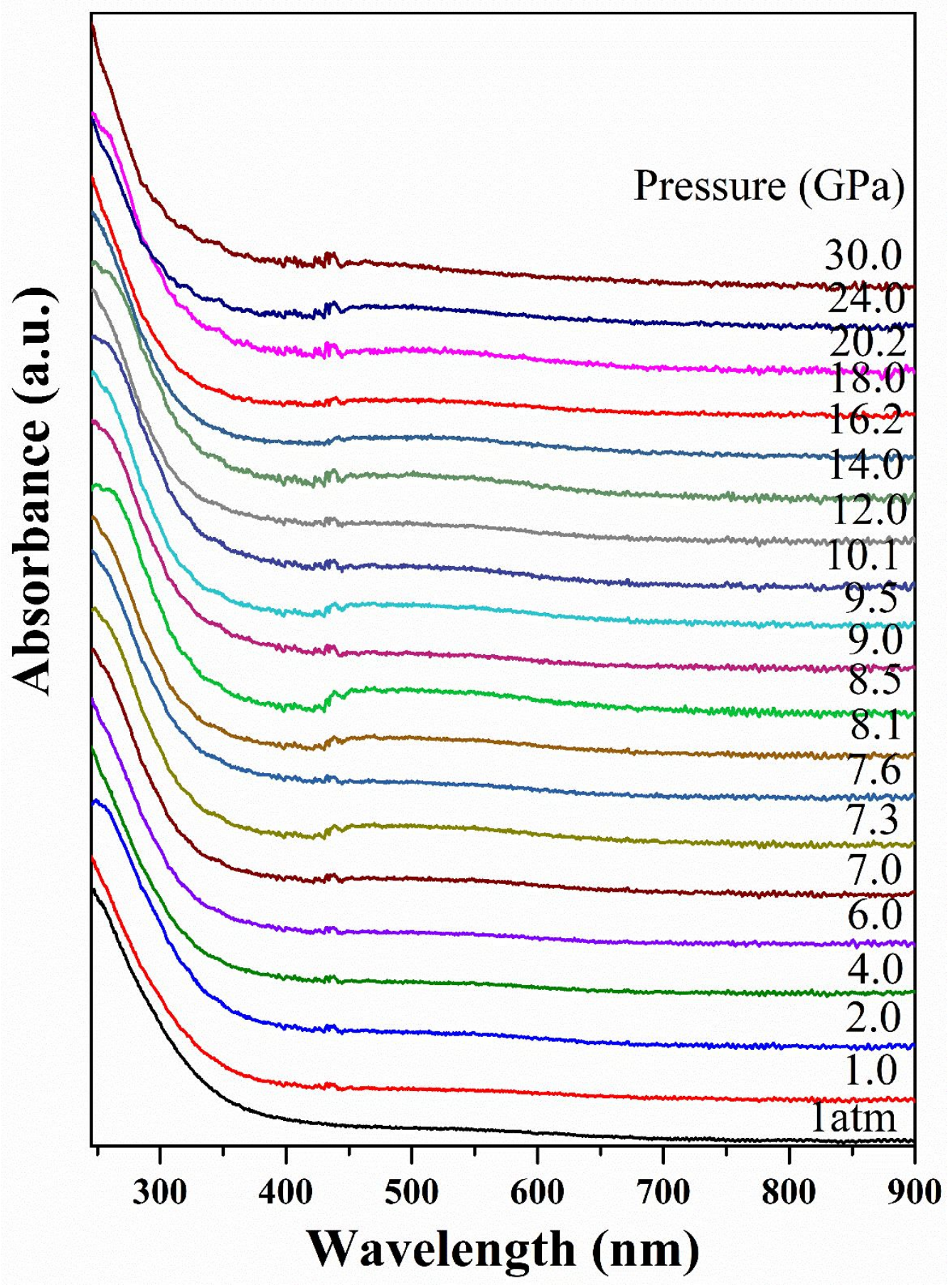

Figure S6. In situ UV-Vis absorption spectra of MTO-0.1 ML during compression. 


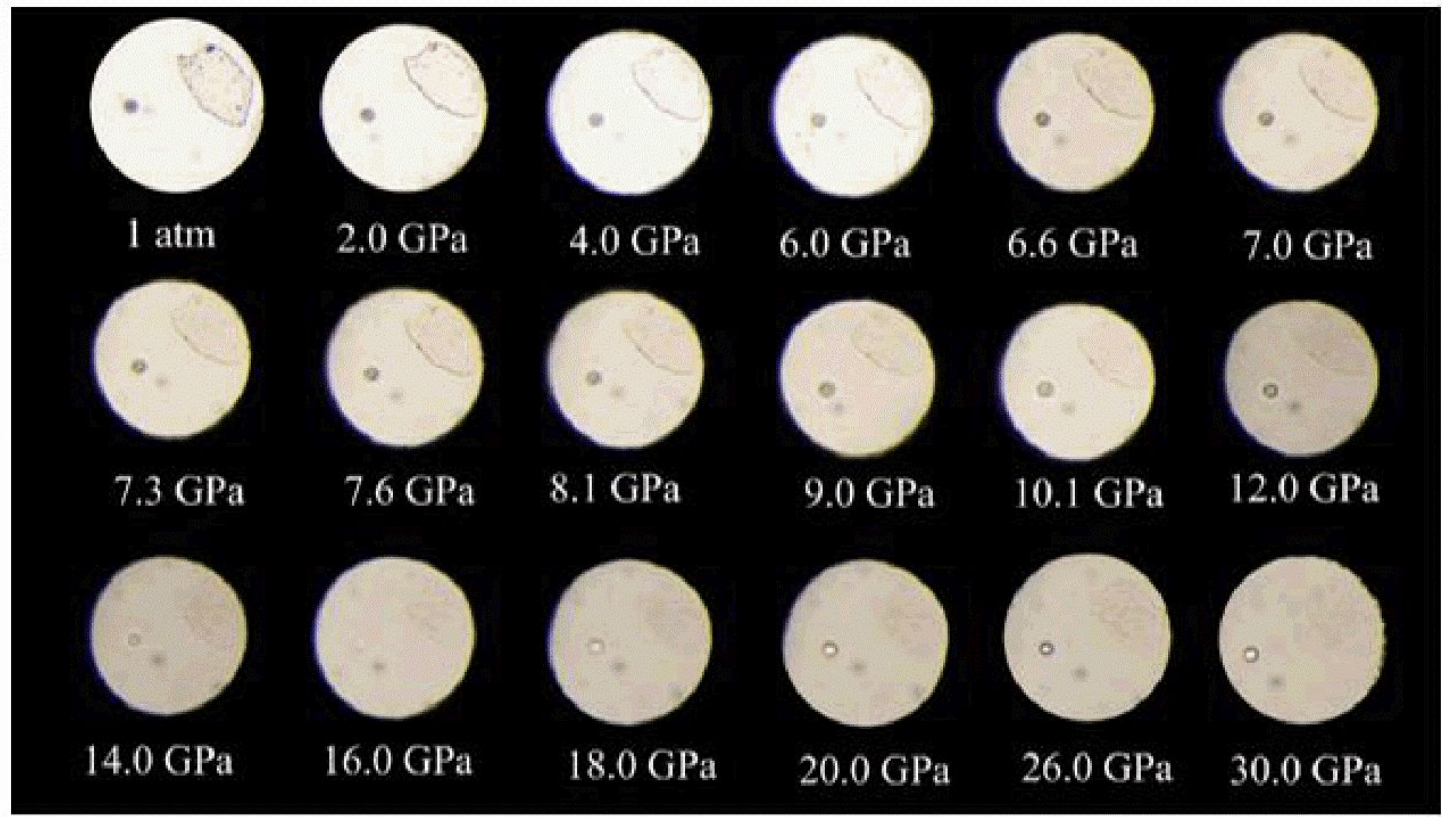

Figure S7. The photos of MTO-0.1ML in DAC upon pressing. 


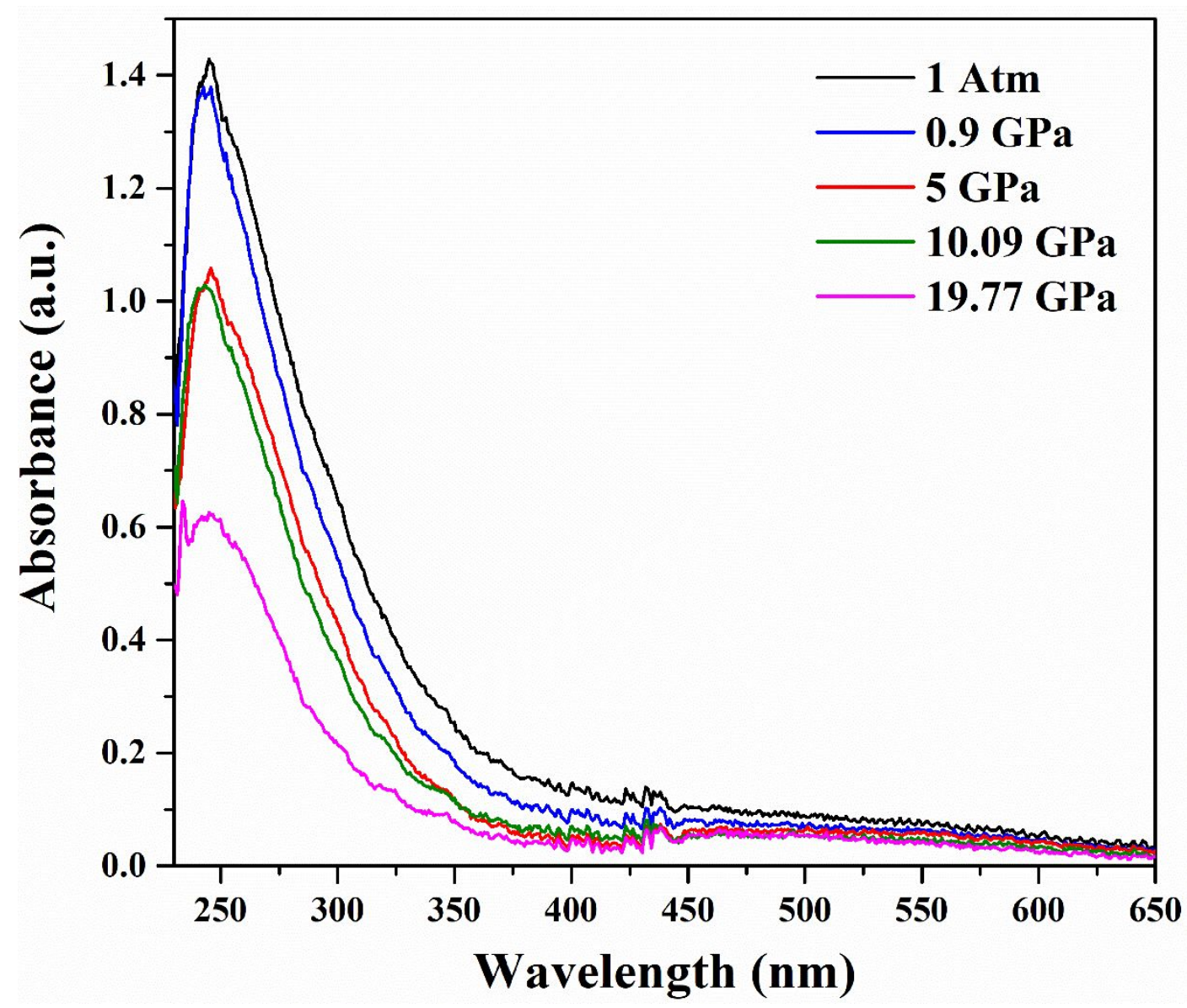

Figure S8. In situ UV-Vis absorption spectra of MTO-0.1ML during decompression. 


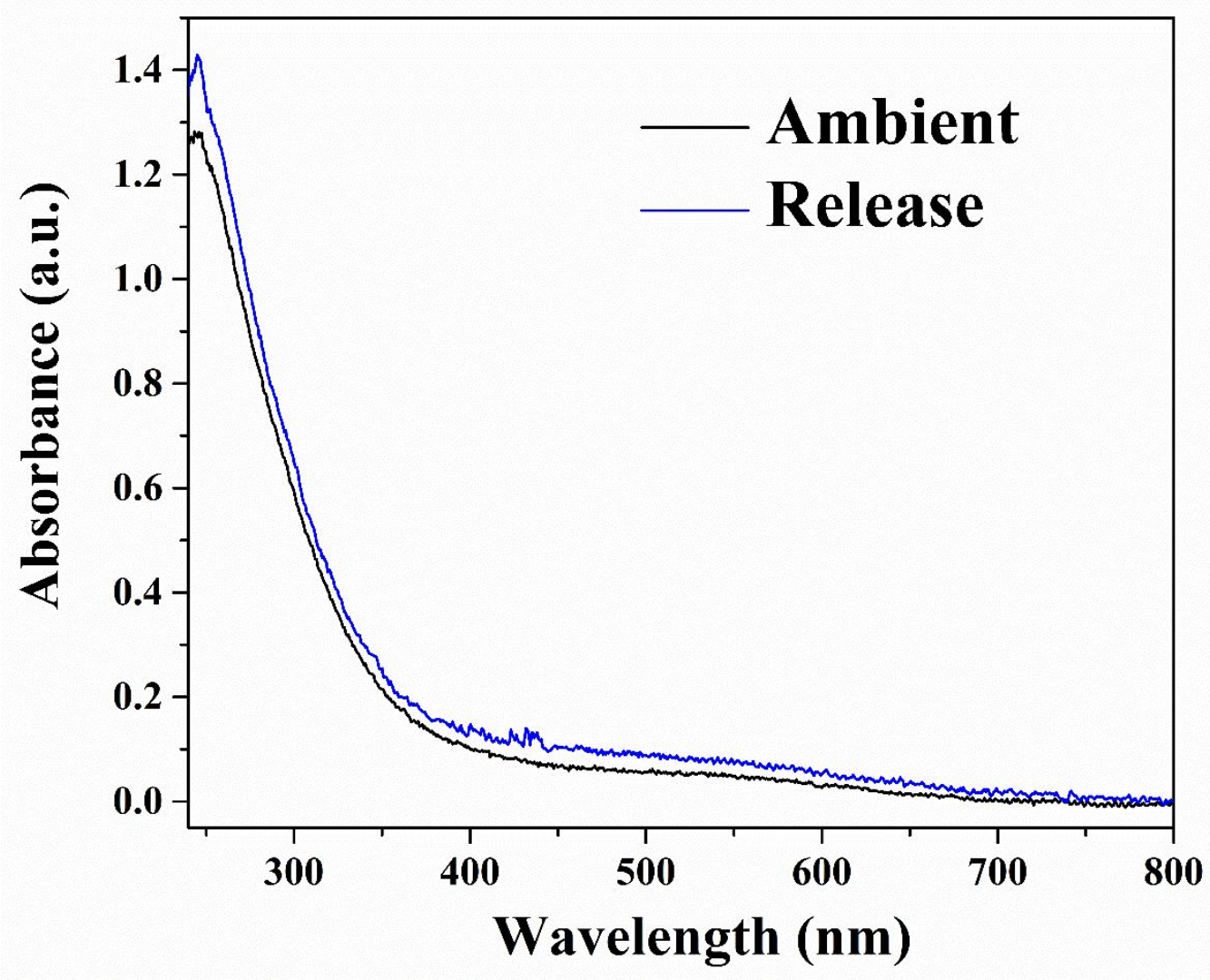

Figure S9. UV-Vis absorption spectra of MTO-0.1ML before and after high-pressure treatment up to $30 \mathrm{GPa}$. 


\section{SUPPLEMENTARY TABLES}

Table S1. Comparison of the color evolution of samples prepared from different raw materials under the same synthesis condition $\left(800^{\circ} \mathrm{C}, 6 \mathrm{~h}\right)$.

\begin{tabular}{|c|c|c|c|c|c|}
\hline \multicolumn{4}{|c|}{ Raw Materials } & \multirow{2}{*}{$\begin{array}{l}\text { Ratio } \\
2.9: 1: 0.1\end{array}$} & \multirow{2}{*}{$\begin{array}{l}\text { Color } \\
6\end{array}$} \\
\hline $\mathrm{ZnO}$ & $\mathrm{TeO}_{2}$ & $\mathrm{MnCO}_{3}$ & & & \\
\hline $\mathrm{ZnO}$ & $\mathrm{TeO}_{2}$ & $\mathrm{Mn}_{2} \mathrm{O}_{3}$ & & $2.9: 1: 0.05$ & \\
\hline $\mathrm{ZnO}$ & $\mathrm{TeO}_{2}$ & $\mathrm{MnO}_{2}$ & & $2.9: 1: 0.1$ & \\
\hline $\mathrm{ZnO}$ & $\mathrm{Te}(\mathrm{OH})_{6}$ & $\mathrm{Mn}_{2} \mathrm{O}_{3}$ & & $2.9: 1: 0.05$ & \\
\hline \multicolumn{2}{|c|}{ Raw Materials } & & & Ratio & Color \\
\hline $\mathrm{MgO}$ & $\mathrm{TeO}_{2}$ & $\mathrm{MnCO}_{3}$ & & $2.9: 1: 0.1$ & \\
\hline $\mathrm{MgO}$ & $\mathrm{TeO}_{2}$ & $\mathrm{Mn}_{2} \mathrm{O}_{3}$ & & $2.9: 1: 0.05$ & \\
\hline $\mathrm{MgO}$ & $\mathrm{TeO}_{2}$ & $\mathrm{MnO}_{2}$ & & $2.9: 1: 0.1$ & \\
\hline $\mathrm{MgO}$ & $\mathrm{Te}(\mathrm{OH})_{6}$ & $\mathrm{Mn}_{2} \mathrm{O}_{3}$ & & $2.9: 1: 0.05$ & \\
\hline \multicolumn{2}{|c|}{ Raw Materials } & & & Ratio & Color \\
\hline $\mathrm{ZnO}$ & $\mathrm{TeO}_{2}$ & $\mathrm{MnCO}_{3}$ & $\mathrm{Li}_{2} \mathrm{CO}_{3}$ & $2.8: 1: 0.1: 0.05$ & \\
\hline $\mathrm{ZnO}$ & $\mathrm{TeO}_{2}$ & $\mathrm{Mn}_{2} \mathrm{O}_{3}$ & $\mathrm{Li}_{2} \mathrm{CO}_{3}$ & $2.8: 1: 0.05: 0.05$ & \\
\hline $\mathrm{ZnO}$ & $\mathrm{TeO}_{2}$ & $\mathrm{MnO}_{2}$ & $\mathrm{Li}_{2} \mathrm{CO}_{3}$ & $2.8: 1: 0.1: 0.05$ & \\
\hline $\mathrm{ZnO}$ & $\mathrm{Te}(\mathrm{OH})_{6}$ & $\mathrm{Mn}_{2} \mathrm{O}_{3}$ & $\mathrm{Li}_{2} \mathrm{CO}_{3}$ & $2.8: 1: 0.05: 0.05$ & \\
\hline \multicolumn{2}{|c|}{ Raw Materials } & & & Ratio & Color \\
\hline $\mathrm{MgO}$ & $\mathrm{TeO}_{2}$ & $\mathrm{MnCO}_{3}$ & $\mathrm{Li}_{2} \mathrm{CO}_{3}$ & $2.8: 1: 0.1: 0.05$ & \\
\hline
\end{tabular}


$\begin{array}{lllll}\mathrm{MgO} & \mathrm{TeO}_{2} & \mathrm{Mn}_{2} \mathrm{O}_{3} & \mathrm{Li}_{2} \mathrm{CO}_{3} & 2.8: 1: 0.05: 0.05\end{array}$

$\begin{array}{lllll}\mathrm{MgO} & \mathrm{TeO}_{2} & \mathrm{MnO}_{2} & \mathrm{Li}_{2} \mathrm{CO}_{3} & 2.8: 1: 0.1: 0.05\end{array}$

$\begin{array}{lllll}\mathrm{MgO} & \mathrm{Te}(\mathrm{OH})_{6} & \mathrm{Mn}_{2} \mathrm{O}_{3} & \mathrm{Li}_{2} \mathrm{CO}_{3} & 2.8: 1: 0.05: 0.05\end{array}$ 
Table S2. The ionic radii $(\AA)$ for related cations with different coordination number.

\begin{tabular}{llll}
\hline Cation & $\mathbf{C N = 4}$ & $\mathbf{C N}=\mathbf{5}$ & $\mathbf{C N}=\mathbf{6}$ \\
\hline $\mathrm{Zn}^{2+}$ & 0.60 & 0.68 & 0.74 \\
$\mathrm{Mg}^{2+}$ & 0.57 & 0.66 & 0.72 \\
$\mathrm{Mn}^{2+}$ & 0.66 & 0.75 & $0.67^{\mathrm{i} / 0.83^{\mathrm{ii}}}$ \\
$\mathrm{Mn}^{3+}$ & - & 0.58 & $0.58^{\mathrm{i} / 0.645^{\mathrm{ii}}}$ \\
$\mathrm{Li}^{+}$ & 0.59 & - & 0.76 \\
\hline $\mathrm{i}$ low spin; ii high spin & & \\
\hline
\end{tabular}


Table S3. Crystallographic data of $(A=\mathrm{Zn}, \mathrm{Mg}) A \mathrm{TO}, A \mathrm{TO}-0.1 \mathrm{M}$, and $A \mathrm{TO}-0.1 \mathrm{ML}$ refined from the room-temperature PXD data.

\begin{tabular}{|c|c|c|c|c|c|c|}
\hline & $i^{*}$ & $\mathrm{ii}^{*}$ & iii $^{*}$ & iv* & $\mathrm{v}^{*}$ & $\mathrm{vi}^{*}$ \\
\hline S. G. & $C 2 / c$ & $C 2 / c$ & $C 2 / c$ & $R-3$ & $R-3$ & $R-3$ \\
\hline$a(\AA ̊)$ & $14.894(1)$ & $14.914(1)$ & $14.878(1)$ & $8.600(1)$ & $8.607(1)$ & $8.609(1)$ \\
\hline$b(\AA)$ & $8.835(1)$ & $8.839(1)$ & $8.819(1)$ & & & \\
\hline$c(\AA)$ & $10.357(1)$ & $10.371(1)$ & $10.351(1)$ & $10.320(1)$ & $10.330(1)$ & $10.335(1)$ \\
\hline$\beta\left({ }^{\circ}\right)$ & $93.0(1)$ & $92.9(1)$ & 93.1(1) & & & \\
\hline$Z$ & 12 & 12 & 12 & 6 & 6 & 6 \\
\hline$V\left(\AA^{3}\right)$ & $1360.9(1)$ & $1365.3(1)$ & $1356.3(1)$ & $661.1(1)$ & $662.8(1)$ & $663.3(1)$ \\
\hline$R_{\mathrm{wp}}(\%)$ & 4.17 & 4.68 & 3.86 & 3.31 & 4.53 & 3.86 \\
\hline$R_{\mathrm{p}}(\%)$ & 4.73 & 4.04 & 3.48 & 4.67 & 5.41 & 5.18 \\
\hline \multicolumn{7}{|c|}{${ }^{*} \mathrm{i}$, ii, iii, iv, v, and vi denote ZTO, ZTO-0.1M, ZTO-0.1ML, MTO, MTO-0.1M, and } \\
\hline
\end{tabular}


Table S4. Atomic coordinates, occupancies of $A \mathrm{TO}, A \mathrm{TO}-0.1 \mathrm{M}$, and $A \mathrm{TO}-0.1 \mathrm{ML}$.

\section{Crystallographic parameters}

\section{ZTO}

\begin{tabular}{|c|c|c|c|c|c|c|}
\hline Atom & Ox. & $x / a$ & $y / b$ & $z / c$ & Occ. & $B_{\text {iso }}$ \\
\hline Te1 & +6 & $0.163(1)$ & $0.499(1)$ & $0.305(1)$ & 1.00 & $0.5(1)$ \\
\hline $\mathrm{Te} 2$ & +6 & 0 & 0 & 0 & 1.00 & $0.3(1)$ \\
\hline $\mathrm{Zn} 1$ & +2 & $0.163(1)$ & $0.268(1)$ & $0.061(1)$ & 1.00 & $0.4(1)$ \\
\hline $\mathrm{Zn} 2$ & +2 & $0.202(1)$ & $0.135(1)$ & $0.426(1)$ & 1.00 & $0.3(1)$ \\
\hline $\mathrm{Zn} 3$ & +2 & $0.361(1)$ & $0.361(1)$ & $0.242(1)$ & 1.00 & $0.4(1)$ \\
\hline $\mathrm{Zn} 4$ & +2 & $0.527(1)$ & $0.153(1)$ & $0.053(1)$ & 1.00 & $0.4(1)$ \\
\hline Zn5 & +2 & 0 & $0.199(1)$ & 0.25 & 1.00 & $0.3(2)$ \\
\hline $\mathrm{O} 1$ & -2 & $0.065(1)$ & $0.011(2)$ & $0.169(1)$ & 1.00 & $0.9(4)$ \\
\hline $\mathrm{O} 2$ & -2 & $0.062(1)$ & $0.173(2)$ & $0.432(2)$ & 1.00 & $0.9(5)$ \\
\hline $\mathrm{O} 3$ & -2 & $0.100(1)$ & $0.343(2)$ & $0.198(2)$ & 1.00 & $1.8(5)$ \\
\hline $\mathrm{O} 4$ & -2 & $0.245(1)$ & $0.349(2)$ & $0.404(2)$ & 1.00 & $0.9(5)$ \\
\hline O5 & -2 & $0.254(1)$ & $0.484(2)$ & $0.171(1)$ & 1.00 & $1.4(5)$ \\
\hline O6 & -2 & $0.273(1)$ & $0.160(2)$ & $0.090(2)$ & 1.00 & $1.2(5)$ \\
\hline $\mathrm{O} 7$ & -2 & $0.386(1)$ & $0.162(2)$ & $0.309(1)$ & 1.00 & $0.9(5)$ \\
\hline O8 & -2 & $0.428(1)$ & $0.003(3)$ & $0.048(1)$ & 1.00 & $0.9(4)$ \\
\hline O9 & -2 & $0.419(1)$ & $0.335(2)$ & $0.066(2)$ & 1.00 & $0.9(5)$ \\
\hline
\end{tabular}

ZTO-0.1M

\begin{tabular}{|c|c|c|c|c|c|c|}
\hline Atom & Ox. & $x / a$ & $y / b$ & $z / c$ & Occ. & $\boldsymbol{B}_{\text {iso }}$ \\
\hline
\end{tabular}




\begin{tabular}{|c|c|c|c|c|c|c|}
\hline $\mathrm{Te} 1$ & +6 & $0.163(1)$ & $0.499(3)$ & $0.305(1)$ & 1.00 & $0.3(1)$ \\
\hline $\mathrm{Te} 2$ & +6 & 0 & 0 & 0 & 1.00 & $0.3(1)$ \\
\hline II $1^{*}$ & +2 & $0.163(1)$ & $0.267(1)$ & $0.061(1)$ & $0.92 / 0.08(3)$ & $0.3(1)$ \\
\hline II $2^{*}$ & +2 & $0.203(1)$ & $0.137(1)$ & $0.427(1)$ & 1.00 & $0.3(1)$ \\
\hline II $3^{*}$ & +2 & $0.361(1)$ & $0.362(1)$ & $0.242(1)$ & 1.00 & $0.3(1)$ \\
\hline III* & +2 & $0.527(1)$ & $0.151(1)$ & $0.053(1)$ & 1.00 & $0.3(1)$ \\
\hline II $5^{*}$ & +2 & 0 & $0.201(1)$ & 0.25 & $0.92 / 0.08(5)$ & $0.5(2)$ \\
\hline $\mathrm{O} 1$ & -2 & $0.070(1)$ & $0.017(2)$ & $0.160(1)$ & 1.00 & $0.9(4)$ \\
\hline $\mathrm{O} 2$ & -2 & $0.072(1)$ & $0.172(2)$ & $0.437(2)$ & 1.00 & $0.9(6)$ \\
\hline $\mathrm{O} 3$ & -2 & $0.099(1)$ & $0.344(2)$ & $0.203(2)$ & 1.00 & $1.3(5)$ \\
\hline $\mathrm{O} 4$ & -2 & $0.246(1)$ & $0.357(2)$ & $0.404(2)$ & 1.00 & $0.9(5)$ \\
\hline O5 & -2 & $0.254(1)$ & $0.483(2)$ & $0.176(1)$ & 1.00 & $0.9(4)$ \\
\hline O6 & -2 & $0.273(1)$ & $0.166(2)$ & $0.094(2)$ & 1.00 & $0.9(5)$ \\
\hline O7 & -2 & $0.390(1)$ & $0.158(2)$ & $0.311(2)$ & 1.00 & $0.9(5)$ \\
\hline O8 & -2 & $0.427(1)$ & $0.004(3)$ & $0.054(1)$ & 1.00 & $0.9(4)$ \\
\hline O9 & -2 & $0.421(1)$ & $0.338(2)$ & $0.065(2)$ & 1.00 & $0.9(6)$ \\
\hline
\end{tabular}

\section{ZTO-0.1ML}

\begin{tabular}{lllllll} 
Atom & Ox. & $\boldsymbol{x} / \boldsymbol{a}$ & $\boldsymbol{y} / \boldsymbol{b}$ & $\boldsymbol{z} / \boldsymbol{c}$ & $\boldsymbol{O c c}$. & $\boldsymbol{B}_{\text {iso }}$ \\
\hline $\mathrm{Te} 1$ & +6 & $0.164(1)$ & $0.499(1)$ & $0.304(1)$ & 1.00 & $0.5(1)$ \\
$\mathrm{Te} 2$ & +6 & 0 & 0 & 0 & 1.00 & $0.5(1)$ \\
$\mathrm{III}^{*}$ & +2 & $0.163(1)$ & $0.268(1)$ & $0.059(1)$ & $0.96 / 0 / 0.04(1)$ & $0.5(6)$ \\
$\mathrm{III}^{*}$ & +2 & $0.203(1)$ & $0.137(1)$ & $0.429(1)$ & $0.91 / 0 / 0.09(1)$ & $0.5(1)$
\end{tabular}




\begin{tabular}{|c|c|c|c|c|c|c|}
\hline III $3^{*}$ & +2 & $0.359(1)$ & $0.361(1)$ & $0.242(1)$ & $0.89 / 0.11(2) / 0$ & $0.5(1)$ \\
\hline III $4^{*}$ & +2 & $0.527(1)$ & $0.151(1)$ & $0.051(1)$ & $0.98 / 0.02(2) / 0$ & $0.5(1)$ \\
\hline${\text { III } 5^{*}}^{2}$ & +2 & 0 & $0.198(1)$ & 0.25 & $0.95 / 0.04(1) / 0.01(8)$ & $0.5(1)$ \\
\hline $\mathrm{O} 1$ & -2 & $0.065(1)$ & $0.017(1)$ & $0.166(1)$ & 1.00 & $1.0(3)$ \\
\hline $\mathrm{O} 2$ & -2 & $0.072(1)$ & $0.168(1)$ & $0.434(1)$ & 1.00 & $1.0(3)$ \\
\hline $\mathrm{O} 3$ & -2 & $0.095(1)$ & $0.342(1)$ & $0.201(1)$ & 1.00 & $1.0(3)$ \\
\hline $\mathrm{O} 4$ & -2 & $0.242(1)$ & $0.345(1)$ & $0.398(1)$ & 1.00 & $1.0(3)$ \\
\hline O5 & -2 & $0.255(1)$ & $0.481(1)$ & $0.173(1)$ & 1.00 & $1.0(3)$ \\
\hline O6 & -2 & $0.272(1)$ & $0.163(1)$ & $0.097(1)$ & 1.00 & $1.0(3)$ \\
\hline $\mathrm{O} 7$ & -2 & $0.392(1)$ & $0.162(1)$ & $0.298(1)$ & 1.00 & $1.0(3)$ \\
\hline O8 & -2 & $0.426(1)$ & $0.007(2)$ & $0.062(1)$ & 1.00 & $1.0(2)$ \\
\hline O9 & -2 & $0.421(1)$ & $0.337(1)$ & $0.069(1)$ & 1.00 & $1.0(3)$ \\
\hline \multicolumn{7}{|l|}{ МТO } \\
\hline Atom & Ox. & $x / a$ & $y / b$ & $z / c$ & Occ. & $B_{\text {iso }}$ \\
\hline $\mathrm{Te} 1$ & +6 & 0 & 0 & 0 & 1.00 & $0.8(1)$ \\
\hline $\mathrm{Te} 2$ & +6 & 0 & 0 & 0.5 & 1.00 & $0.8(1)$ \\
\hline $\mathrm{O} 1$ & -2 & $0.204(1)$ & $0.173(1)$ & $0.103(1)$ & 1.00 & $1.0(2)$ \\
\hline $\mathrm{O} 2$ & -2 & $0.189(1)$ & $0.164(1)$ & $0.618(1)$ & 1.00 & $1.0(2)$ \\
\hline Mg1 & +2 & $0.401(1)$ & $0.108(1)$ & $0.043(1)$ & 1.00 & $0.8(1)$ \\
\hline
\end{tabular}

\section{MTO.0.1M}

\begin{tabular}{ccccccc} 
Atom & Ox. & $\boldsymbol{x} / \boldsymbol{a}$ & $\boldsymbol{y} / \boldsymbol{b}$ & $\boldsymbol{z} / \boldsymbol{c}$ & $\boldsymbol{O c c .}$ & $\boldsymbol{B}_{\text {iso }}$ \\
\hline $\mathrm{Te} 1$ & +6 & 0 & 0 & 0 & 1.00 & $0.5(1)$
\end{tabular}




\begin{tabular}{|c|c|c|c|c|c|c|}
\hline $\mathrm{Te} 2$ & +6 & 0 & 0 & 0.5 & 1.00 & $0.5(1)$ \\
\hline $\mathrm{O} 1$ & -2 & $0.205(1)$ & $0.175(1)$ & $0.106(1)$ & 1.00 & $1.0(4)$ \\
\hline $\mathrm{O} 2$ & -2 & $0.192(1)$ & $0.165(1)$ & $0.619(1)$ & 1.00 & $1.0(3)$ \\
\hline $\mathrm{V} 1^{*}$ & +2 & $0.400(1)$ & $0.109(1)$ & $0.042(1)$ & $0.97 / 0.03(1)$ & $0.5(1)$ \\
\hline \multicolumn{7}{|c|}{ MTO.0.1ML } \\
\hline Atom & Ox. & $x / a$ & $y / b$ & $z / c$ & Occ. & $B_{\text {iso }}$ \\
\hline Te1 & +6 & 0 & 0 & 0 & 1 & $0.6(1)$ \\
\hline $\mathrm{Te} 2$ & +6 & 0 & 0 & 0.5 & 1 & $0.6(1)$ \\
\hline $\mathrm{O} 1$ & -2 & $0.204(1)$ & $0.175(1)$ & $0.105(1)$ & 1 & $1.1(2)$ \\
\hline $\mathrm{O} 2$ & -2 & $0.192(1)$ & $0.163(1)$ & $0.618(1)$ & 1 & $1.1(2)$ \\
\hline $\mathrm{VV}^{*}$ & $+2 /+3 /+1$ & $0.400(1)$ & $0.109(1)$ & $0.043(1)$ & $0.94 / 0.03(12) / 0.03(9)$ & $0.6(1)$ \\
\hline \multicolumn{7}{|c|}{${ }^{*} \mathrm{II} n, \mathrm{III} n, \mathrm{~V} 1, \mathrm{VV} 1$ represent Znn $n|\mathrm{Mn} n, \mathrm{Zn} n| \mathrm{Mn} n|\operatorname{Li} n, \operatorname{Mg} 1| \mathrm{Mn} 1, \operatorname{Mg} 1|\mathrm{Mn} 1| \operatorname{Li} 1$} \\
\hline
\end{tabular}


Table S5. The bond lengths and angle values of refined samples.

\begin{tabular}{|c|c|c|c|c|c|}
\hline \multirow{2}{*}{$\begin{array}{l}\text { ZTO } \\
\text { Bond }\end{array}$} & \multirow[b]{2}{*}{$d(\AA ̊)$} & \multicolumn{2}{|c|}{ ZTO-0.1M } & \multicolumn{2}{|c|}{ ZTO-0.1ML } \\
\hline & & Bond & $d(\AA ̊)$ & Bond & $d(\AA ̊)$ \\
\hline \multirow[t]{6}{*}{ Te1-O } & $1.974(17)$ & Te1-O & $1.954(18)$ & Te1-O & $1.939(11)$ \\
\hline & $1.979(17)$ & & $1.956(13)$ & & $1.978(9)$ \\
\hline & $1.987(13)$ & & $1.988(17)$ & & $1.978(9)$ \\
\hline & $2.004(17)$ & & $2.011(16)$ & & $1.990(10)$ \\
\hline & $2.044(16)$ & & $2.017(17)$ & & $1.992(10)$ \\
\hline & $2.090(13)$ & & $2.042(14)$ & & $2.017(11)$ \\
\hline \multirow[t]{3}{*}{ Te2-O } & $1.937(18) \times 2$ & Te2-O & $1.917(15) \times 2$ & Te2-O & $1.935(8) \times 2$ \\
\hline & $1.959(13) \times 2$ & & $1.990(19) \times 2$ & & $1.976(12) \times 2$ \\
\hline & $2.035(17) \times 2$ & & $1.992(19) \times 2$ & & $2.008(12) \times 2$ \\
\hline \multirow[t]{4}{*}{ Zn1-O } & $1.861(18)$ & $\mathrm{II} 1^{*}-\mathrm{O}$ & $1.887(16)$ & III $1^{*}-\mathrm{O}$ & $1.884(10)$ \\
\hline & $1.909(17)$ & & $1.920(19)$ & & $1.942(11)$ \\
\hline & $1.966(17)$ & & $1.991(20)$ & & $2.004(11)$ \\
\hline & $1.973(19)$ & & $1.996(18)$ & & $2.013(11)$ \\
\hline \multirow[t]{4}{*}{$\mathrm{Zn} 2-\mathrm{O}$} & $1.815(16)$ & II $2 *-O$ & $1.859(16)$ & III $2 *-O$ & $1.860(11)$ \\
\hline & $1.896(17)$ & & $1.873(17)$ & & $1.928(10)$ \\
\hline & $2.020(18)$ & & $1.990(20)$ & & $1.959(13)$ \\
\hline & $2.108(17)$ & & $2.065(18)$ & & $1.972(11)$ \\
\hline \multirow[t]{2}{*}{$\mathrm{Zn} 3-\mathrm{O}$} & $1.917(18)$ & $\mathrm{II} 3 *-\mathrm{O}$ & $1.969(17)$ & III $3 *-O$ & $1.901(12)$ \\
\hline & $1.926(16)$ & & $1.982(18)$ & & $1.979(10)$ \\
\hline
\end{tabular}




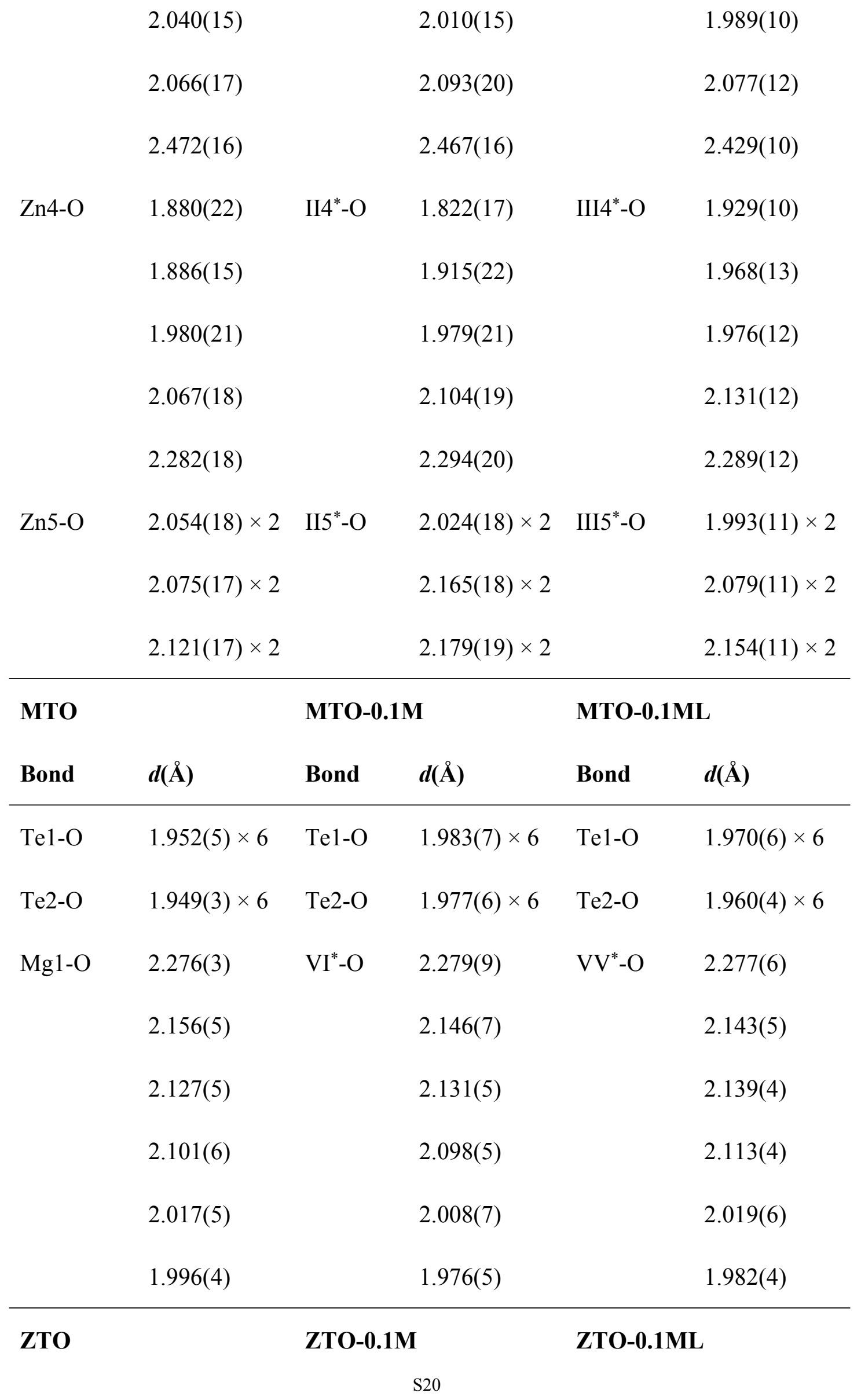




\begin{tabular}{|c|c|c|c|c|c|}
\hline & Angle( $\left(^{\circ}\right)$ & & Angle( $\left.{ }^{\circ}\right)$ & & Angle ( $\left.{ }^{\circ}\right)$ \\
\hline \multirow[t]{15}{*}{ O-Te1-O } & $96.6(3)$ & O-Te1-O & $99.0(3)$ & O-Te1-O & $95.0(4)$ \\
\hline & $96.2(3)$ & & $96.4(7)$ & & $94.8(4)$ \\
\hline & $95.0(3)$ & & $94.9(3)$ & & $94.1(4)$ \\
\hline & $92.2(3)$ & & $94.4(3)$ & & $93.5(4)$ \\
\hline & $91.4(3)$ & & $93.2(3)$ & & $93.4(4)$ \\
\hline & $87.4(3)$ & & $89.8(3)$ & & $92.1(4)$ \\
\hline & $86.1(3)$ & & $87.7(3)$ & & $89.2(4)$ \\
\hline & $84.1(3)$ & & $86.1(3)$ & & $88.5(4)$ \\
\hline & $84.0(3)$ & & $85.6(3)$ & & $86.3(4)$ \\
\hline & $83.3(3)$ & & $85.3(3)$ & & $85.4(4)$ \\
\hline & $82.7(3)$ & & $85.0(3)$ & & $85.3(4)$ \\
\hline & $178.8(3)$ & & $82.9(3)$ & & $82.7(4)$ \\
\hline & 176.3(3) & & $177.5(3)$ & & $177.3(4)$ \\
\hline & $164.6(3)$ & & $176.0(3)$ & & 177.1(3) \\
\hline & $101.0(3)$ & & $165.9(3)$ & & $169.2(4)$ \\
\hline \multirow[t]{6}{*}{$\mathrm{O}-\mathrm{Te} 2-\mathrm{O}$} & 180.0 & O-Te2-O & 180 & $\mathrm{O}-\mathrm{Te} 2-\mathrm{O}$ & 180 \\
\hline & $82.0(3)$ & & $84.2(7)$ & & $83.7(4)$ \\
\hline & $82.2(3)$ & & $85.9(8)$ & & $85.6(5)$ \\
\hline & $89.2(3)$ & & $86.1(8)$ & & $89.3(4)$ \\
\hline & $90.8(3)$ & & $93.9(8)$ & & $90.7(4)$ \\
\hline & $97.8(3)$ & & $94.1(8)$ & & $94.4(5)$ \\
\hline
\end{tabular}




\begin{tabular}{|c|c|c|c|c|c|}
\hline & $98.0(3)$ & & $95.8(7)$ & & $96.4(4)$ \\
\hline \multirow[t]{6}{*}{$\mathrm{O}-\mathrm{Zn} 1-\mathrm{O}$} & $85.8(7)$ & O-III ${ }^{*}-\mathrm{O}$ & $85.4(8)$ & $\mathrm{O}-\mathrm{III} 1^{*}-\mathrm{O}$ & $118.9(4)$ \\
\hline & $80.8(7)$ & & $81.0(7)$ & & $114.6(4)$ \\
\hline & $140.2(8)$ & & $142.0(8)$ & & $82.8(4)$ \\
\hline & $121.5(8)$ & & $119.2(8)$ & & $108.7(5)$ \\
\hline & $111.8(7)$ & & $114.6(8)$ & & $142.4(4)$ \\
\hline & $110.8(8)$ & & $110.2(8)$ & & $85.5(4)$ \\
\hline \multirow[t]{6}{*}{$\mathrm{O}-\mathrm{Zn} 2-\mathrm{O}$} & $84.9(3)$ & $\mathrm{O}-\mathrm{II} 2^{*}-\mathrm{O}$ & $115.1(7)$ & $\mathrm{O}-\mathrm{III} 2^{*}-\mathrm{O}$ & $116.6(4)$ \\
\hline & $121.6(3)$ & & $120.5(7)$ & & $119.6(5)$ \\
\hline & 115.1(3) & & $120.5(8)$ & & $118.8(5)$ \\
\hline & $108.7(3)$ & & $107.5(7)$ & & $87.7(5)$ \\
\hline & $119.8(3)$ & & $88.0(7)$ & & $108.9(4)$ \\
\hline & $100.2(3)$ & & $99.8(7)$ & & $100.3(5)$ \\
\hline \multirow[t]{10}{*}{$\mathrm{O}-\mathrm{Zn} 3-\mathrm{O}$} & $98.1(3)$ & O-II $3^{*}-\mathrm{O}$ & $110.4(7)$ & O-III3*-O & $141.7(5)$ \\
\hline & $95.8(3)$ & & $101.0(7)$ & & $111.3(5)$ \\
\hline & $95.5(3)$ & & $107.0(7)$ & & $92.6(5)$ \\
\hline & $80.8(3)$ & & $91.2(6)$ & & $85.8(4)$ \\
\hline & $72.8(3)$ & & $139.6(7)$ & & $102.8(4)$ \\
\hline & $158.6(3)$ & & $97.5(7)$ & & $96.7(4)$ \\
\hline & $138.6(3)$ & & $83.2(6)$ & & $72.8(3)$ \\
\hline & $111.4(3)$ & & $96.9(7)$ & & $102.2(4)$ \\
\hline & $104.4(3)$ & & $71.0(5)$ & & $97.5(4)$ \\
\hline & & & S22 & & \\
\hline
\end{tabular}




\begin{tabular}{|c|c|c|c|c|c|}
\hline & $102.7(3)$ & & $160.0(7)$ & & $159.4(4)$ \\
\hline \multirow[t]{10}{*}{$\mathrm{O}-\mathrm{Zn} 4-\mathrm{O}$} & $73.5(3)$ & $\mathrm{O}-\mathrm{II} 4^{*}-\mathrm{O}$ & $103.2(7)$ & $\mathrm{O}-\mathrm{III} 4^{*}-\mathrm{O}$ & $107.1(4)$ \\
\hline & $134.2(3)$ & & $119.7(7)$ & & $115.5(4)$ \\
\hline & $95.5(3)$ & & $101.5(8)$ & & $102.7(4)$ \\
\hline & $103.6(3)$ & & $112.2(8)$ & & $107.4(4)$ \\
\hline & $87.0(3)$ & & $79.7(8)$ & & $82.7(5)$ \\
\hline & $145.6(3)$ & & $93.7(8)$ & & $93.6(5)$ \\
\hline & $112.9(3)$ & & $144.2(7)$ & & $145.3(5)$ \\
\hline & $77.6(3)$ & & $138.7(8)$ & & $141.0(5)$ \\
\hline & $122.2(3)$ & & $87.2(7)$ & & $85.7(4)$ \\
\hline & $101.3(3)$ & & $74.6(7)$ & & $75.5(4)$ \\
\hline \multirow[t]{9}{*}{$\mathrm{O}-\mathrm{Zn} 5-\mathrm{O}$} & $103.5(3)$ & $\mathrm{O}-\mathrm{II} 5^{*}-\mathrm{O}$ & $102.5(7)$ & O-III5 ${ }^{*}-\mathrm{O}$ & $100.8(4)$ \\
\hline & $90.7(3)$ & & $89.5(7)$ & & $91.7(4)$ \\
\hline & $91.5(3)$ & & $161.0(7)$ & & $161.8(4)$ \\
\hline & $97.2(3)$ & & $87.5(7)$ & & $88.6(4)$ \\
\hline & $160.8(3)$ & & $100.9(7)$ & & $100.5(4)$ \\
\hline & $75.2(3)$ & & $83.1(6)$ & & $79.7(4)$ \\
\hline & $76.9(2)$ & & $94.1(6)$ & & $93.0(4)$ \\
\hline & $94.7(3)$ & & $75.7(6)$ & & $76.6(4)$ \\
\hline & $167.3(3)$ & & $166.6(7)$ & & $165.9(4)$ \\
\hline \multirow[t]{2}{*}{ MTO } & & \multicolumn{2}{|c|}{ MTO-0.1M } & \multicolumn{2}{|c|}{ MTO-0.1ML } \\
\hline & Angle( $\left(^{0}\right)$ & & Angle( $\left(^{\circ}\right)$ & & Angle( $\left.{ }^{\circ}\right)$ \\
\hline
\end{tabular}




\begin{tabular}{|c|c|c|c|c|c|}
\hline \multirow[t]{3}{*}{ O-Te1-O } & $93.1(2)$ & O-Te1-O & $92.4(3)$ & O-Te1-O & $92.7(2)$ \\
\hline & $86.9(2)$ & & $87.6(3)$ & & $87.3(2)$ \\
\hline & 180 & & 180 & & 180 \\
\hline \multirow[t]{3}{*}{$\mathrm{O}-\mathrm{Te} 2-\mathrm{O}$} & $85.4(2)$ & $\mathrm{O}-\mathrm{Te} 2-\mathrm{O}$ & $85.5(3)$ & O-Te2-O & $85.3(2)$ \\
\hline & $94.6(2)$ & & $94.5(3)$ & & $94.7(2)$ \\
\hline & 180 & & 180 & & 180 \\
\hline \multirow[t]{15}{*}{ O-Mg1-O } & $95.4(2)$ & $\mathrm{O}-\mathrm{V} 1{ }^{*}-\mathrm{O}$ & $94.7(3)$ & $\mathrm{O}-\mathrm{VV} 1^{*}-\mathrm{O}$ & $94.8(2)$ \\
\hline & $92.6(2)$ & & $93.0(3)$ & & $92.7(2)$ \\
\hline & $81.0(2)$ & & $80.8(3)$ & & $80.9(2)$ \\
\hline & $79.6(2)$ & & $79.2(3)$ & & $79.7(2)$ \\
\hline & $79.3(2)$ & & $78.7(3)$ & & $79.5(2)$ \\
\hline & $79.2(2)$ & & $78.4(3)$ & & $78.2(2)$ \\
\hline & $76.8(2)$ & & $77.9(3)$ & & $77.3(2)$ \\
\hline & $75.0(2)$ & & $76.9(3)$ & & $76.0(2)$ \\
\hline & $157.7(2)$ & & $159.0(3)$ & & $158.1(3)$ \\
\hline & $151.1(2)$ & & $151.6(4)$ & & $151.0(3)$ \\
\hline & $145.4(2)$ & & $145.8(2)$ & & $146.0(2)$ \\
\hline & $122.5(2)$ & & $122.9(4)$ & & $123.2(3)$ \\
\hline & $107.2(2)$ & & 106.7(3) & & $106.6(2)$ \\
\hline & $106.3(2)$ & & $106.2(3)$ & & $106.4(2)$ \\
\hline & $105.2(2)$ & & $104.7(3)$ & & $105.0(2)$ \\
\hline
\end{tabular}


*IIn, III $n, \mathrm{~V} 1$, VV1 represent Znn $|\mathrm{Mn} n, \mathrm{Zn} n| \operatorname{Mn} n|\operatorname{Li} n, \operatorname{Mg} 1| \operatorname{Mn} 1, \operatorname{Mg} 1|\operatorname{Mn} 1| \operatorname{Li} 1$ ( $n=$ $1,2,3,4,5)$. 
Table S6. The CIE $L^{*} a^{*} b^{*}$ values of the samples after chemical and thermal stability examination.

\begin{tabular}{|c|c|c|c|c|c|}
\hline Sample & & $L^{*}$ & $a^{*}$ & $b^{*}$ & $\Delta \boldsymbol{E}^{*}$ \\
\hline \multirow[t]{5}{*}{ ZTO-0.1M } & water & +81.90 & +3.15 & +23.50 & - \\
\hline & $4 \% \mathrm{NaOH}$ & +81.21 & +3.02 & +23.85 & 0.95 \\
\hline & $4 \% \mathrm{HNO}_{3}$ & +82.15 & +2.95 & +23.42 & 0.33 \\
\hline & $4 \% \mathrm{HCl}$ & - & - & - & - \\
\hline & $800^{\circ} \mathrm{C}$ & +81.86 & +3.17 & +23.45 & 0.07 \\
\hline \multirow[t]{5}{*}{ ZTO-0.1ML } & water & +50.22 & +4.49 & -9.26 & - \\
\hline & $4 \% \mathrm{NaOH}$ & +50.16 & +4.32 & -8.95 & 0.36 \\
\hline & $4 \% \mathrm{HNO}_{3}$ & - & - & - & - \\
\hline & $4 \% \mathrm{HCl}$ & - & - & - & - \\
\hline & $900{ }^{\circ} \mathrm{C}$ & +50.81 & +4.20 & -8.71 & 0.86 \\
\hline \multirow[t]{5}{*}{ MTO-0.1ML } & water & +58.98 & +6.20 & -15.54 & - \\
\hline & $4 \% \mathrm{NaOH}$ & +58.61 & +6.15 & -14.96 & 0.69 \\
\hline & $4 \% \mathrm{HNO}_{3}$ & +59.26 & +5.98 & -14.75 & 0.87 \\
\hline & $4 \% \mathrm{HCl}$ & +59.50 & +5.87 & -14.66 & 1.07 \\
\hline & $900{ }^{\circ} \mathrm{C}$ & +58.30 & +6.32 & -15.21 & 0.77 \\
\hline
\end{tabular}


Table S7. Crystallographic data, atomic coordinates, and occupancies of HP-MTO0.1 ML refined from room-temperature PXD data.

\begin{tabular}{|c|c|c|c|c|c|c|}
\hline Atom & Ox. & $x / a$ & $y / b$ & $z / c$ & Occ. & $B_{\text {iso }}$ \\
\hline $\mathrm{Mg} 1$ & +2 & 0 & 0 & $0.007(1)$ & 1 & $0.5(1)$ \\
\hline $\operatorname{Mg} 2|\operatorname{Mn} 2| \operatorname{Li} 2$ & $+2|+3|+1$ & 0 & 0 & $0.218(1)$ & $0.8 / 0.1 / 0.1(1)$ & $0.5(1)$ \\
\hline $\mathrm{Mg} 3$ & +2 & 0 & 0 & $0.498(1)$ & 1 & $0.5(1)$ \\
\hline $\mathrm{Te} 1$ & +6 & 0 & 0 & $0.708(1)$ & 1 & $0.5(1)$ \\
\hline $\mathrm{O} 1$ & -2 & $0.325(2)$ & $0.045(2)$ & $0.115(1)$ & 1 & $0.8(3)$ \\
\hline $\mathrm{O} 2$ & -2 & $0.354(2)$ & $0.369(2)$ & $0.292(1)$ & 1 & $0.9(4)$ \\
\hline \multicolumn{7}{|c|}{ S.G. $R 3 ; a=5.159(1) \AA ; \mathrm{c}=13.855(1) \AA ; V=319.3(1) \AA^{3} ; R_{\mathrm{wp}}=6.31 \%, R_{\mathrm{p}}=$} \\
\hline $4.85 \%$. & & & & & & \\
\hline
\end{tabular}


Table S8. Selected bond lengths and angles of HP-MTO-0.1ML.

\begin{tabular}{|c|c|c|c|}
\hline \multicolumn{2}{|c|}{ Bond(Å) } & \multicolumn{2}{|r|}{ Angle( $\left(^{\circ}\right)$} \\
\hline \multirow[t]{2}{*}{ Mg1-O } & $1.810(7) \times 3$ & $\mathrm{O}-\mathrm{Te}-\mathrm{O}$ & $168.5(2)$ \\
\hline & $2.165(8) \times 3$ & & $96.2(2)$ \\
\hline \multirow[t]{2}{*}{ Mg2|Mn2|Li2-O } & $2.159(7) \times 3$ & & $94.4(2)$ \\
\hline & $2.106(8) \times 3$ & & $90.0(2)$ \\
\hline \multirow[t]{2}{*}{$\mathrm{Mg} 3-\mathrm{O}$} & $2.393(8) \times 3$ & & $80.3(2)$ \\
\hline & $2.096(6) \times 3$ & $\mathrm{O}-\mathrm{Mg} 1-\mathrm{O}$ & $154.6(3)$ \\
\hline \multirow[t]{14}{*}{$\mathrm{Te}-\mathrm{O}$} & $1.948(8) \times 3$ & & $107.6(3)$ \\
\hline & $1.951(6) \times 3$ & & $94.4(2)$ \\
\hline & & & $93.5(2)$ \\
\hline & & & $77.9(3)$ \\
\hline & & & $77.0(2)$ \\
\hline & & $\mathrm{O}-\mathrm{Mg} 2|\mathrm{Mn} 2| \mathrm{Li} 2-\mathrm{O}$ & $165.6(3)$ \\
\hline & & & $98.8(3)$ \\
\hline & & & $93.4(2)$ \\
\hline & & & $86.8(2)$ \\
\hline & & & $79.6(2)$ \\
\hline & & $\mathrm{O}-\mathrm{Mg} 3-\mathrm{O}$ & $150.4(3)$ \\
\hline & & & $110.2(2)$ \\
\hline & & & $87.3(2)$ \\
\hline & & & $84.0(2)$ \\
\hline
\end{tabular}


70.4(2) 


\begin{tabular}{ll}
\hline Table S9. Crystallographic Data and Details of the Structure Refinement of HP- \\
MTO-01ML \\
\hline Chemical formula & $\mathrm{Mg}_{2.8} \mathrm{Mn}_{0.1} \mathrm{Li}_{0.1} \mathrm{TeO}_{6}$ \\
Formula weight & 297.8 \\
Temperature & $298 \mathrm{~K}$ \\
Radiation & synchrotron \\
Wavelength & $0.68993 \AA$ \\
Crystal system & trigonal \\
Space group (No.) & $R 3(146)$ \\
Cell parameters & $a=5.1587(1) \AA, c=13.8548(1) \AA$ \\
$V$ & $319.3(1) \AA 3$ \\
$Z$ & $6.31 \%$ \\
$R_{\text {wp }}$ & $1-57^{3}$ \\
\hline range & $4.85 \%$ \\
\hline
\end{tabular}

